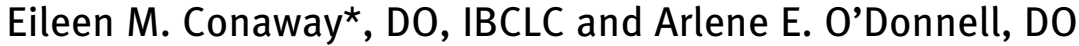 Osteopathic considerations for breastfeeding women
}

https://doi.org/10.1515/jom-2021-0069

Received February 28, 2021; accepted June 3, 2021;

published online July 14, 2021

\begin{abstract}
Extensive benefits of breastfeeding have been identified and although many women initiate breastfeeding, discontinuation is common when problems arise. In this article, the authors review the pertinent anatomy of the breast with osteopathic considerations to help osteopathic physicians identify myofascial, vascular, lymphatic, or innervation somatic dysfunctions to support the breastfeeding mothers' long term breastfeeding goals, optimize breastmilk supply, and potentially prevent or treat problems that could interfere with lactation. Observations from the authors' academic mother-baby osteopathic manipulation clinic are also included.
\end{abstract}

Keywords: breast anatomy; breastfeeding; infants; osteopathic manipulation; pregnancy.

The short- and long-term benefits of breastfeeding to both the mother and infant are well established and include, for the child, protection against common childhood infections $[1,2]$, the prevention of type-2 diabetes and systolic hypertension [1], lower rates of sudden infant death syndrome [2], increased performance on intelligence tests [1], reduction in obesity [2], and favorable intestinal microbiota [3]. Maternal benefits include a reduction in the risks of breast cancer, diabetes, hyperlipidemia, hypertension, myocardial infarction, obesity, and ovarian cancer [4]. Breastfeeding also provides optimal infant nutrition and other nonnutritive benefits [5]. Given this evidence, the American Academy of Pediatrics [6], and the Centers for

*Corresponding author: Eileen M. Conaway, DO, IBCLC, Tidelands Health Family Medicine, Holmestown Road, 4320 Holmestown Road, Myrtle Beach, SC 29588-7837, USA; and AdventHealth Family Medicine Residency in East Orlando, Orlando, FL, USA, E-mail: econawaydo@gmail.comhttps://orcid.org/0000-0003-34194889

Arlene E. O'Donnell, DO, AdventHealth Family Medicine Residency in East Orlando, Orlando, FL, USA. https://orcid.org/0000-0002-52514316
Disease Control (CDC) [6] recommend exclusive breastfeeding of infants for the first 6 months and continued breastfeeding for 1 year. The World Health Organization [5] recommends exclusive breastfeeding for 6 months and continued breastfeeding with complimentary foods to 2 years and beyond. According to CDC data based on United States births in 2017, $84.1 \%$ of women initiated breastfeeding at birth but only $58.3 \%$ continued at 6 months with just $25.6 \%$ doing so exclusively [7]. A 2010 review article [8] in the osteopathic literature regarding breastfeeding revealed a focus on the treatment of infant somatic dysfunction when breastfeeding difficulties are encountered [8]. Continued osteopathic care of the breastfeeding mother could be vital to achieving a long-term breastfeeding relationship. The osteopathic physician could potentially address maternal issues with breastfeeding, including breast and nipple pain, low milk supply, clogged milk ducts, and mastitis. To that end, we present a review of relevant anatomy and osteopathic techniques that can be extrapolated to the treatment of the breastfeeding mother as well as observations from our academic mother-baby osteopathic manipulation clinic.

\section{Clinical summary}

\section{Lactogenesis}

A general understanding of the process of breastmilk production may help physicians provide better care to their breastfeeding patients. The breast is a secretory organ under hormonal control in the initial stages of lactogenesis. Lactogenesis I occurs between 16 and 22 weeks gestation, when the anterior pituitary increases secretion of prolactin [9]. This causes breast cell differentiation and remodeling to prepare for lactation [9]. Between 2 and 5 days after delivery, milk production begins in earnest with lactogenesis II [9]. This stage is triggered by a marked decrease in progesterone after placental delivery [9]. The hallmark of this stage is physiologic postpartum engorgement and is colloquially known as the "milk coming in." [9] Lactogenesis III, mature milk production, begins around postpartum day 9 and continues until the cessation of 
breastfeeding [9]. Until this point, breastmilk production is a hormonally driven endocrine process, but with the transition to lactogenesis III, it becomes an autocrine process based almost entirely on supply and demand [9]. Oxytocin, produced in the posterior pituitary gland, plays a role in milk letdown even during the largely autocrine process of lactogenesis III [9].

\section{Myofascial}

"The fascia is the ground in which all causes of death do the destruction of life." - A.T. Still [10]

The breast is positioned anterior to the chest wall musculature between ribs 2-6 vertically and between the sternum and midaxillary line horizontally. Posterior to the breast tissue, a deep layer of fascia separates it from the fascia covering the pectoralis major, serratus anterior, rectus abdominus, and external oblique muscles, creating a retromammary space [11, 12]. This space is filled with loose connective tissue, which allows breast motion independent of the chest wall [11]. While no specific osteopathic technique has been described for mobilizing the breast tissue, applying biomechanical myofascial release as described in Foundations of Osteopathic Medicine [13] can attain balanced motion of the breast just as it can for any other structure.

Superficially, the breast is separated from the skin by the superficial fascial layer, which is organized superiorly to rib $4[11,12]$. An array of transverse ligaments spread out from the periosteum of rib 5 to the dermis to provide support for the breast [12]. Based on anatomical dissections, rib 5 may be the key rib to assess osteopathically as it relates to the breast and can be treated using a ligamentous articular strain (LAS) technique or another preferred technique if dysfunction is identified [11, 14]. In our osteopathic manipulation clinic, we frequently observe somatic dysfunctions of ribs 3 through 5 in women with lactation concerns. Additionally, this section of ribs often becomes dysfunctional in the late second or early third trimester due both to increasing breast size (resulting in a change in postural fulcrum) and the onset of lactogenesis I. When prenatal patients present with pain and a corresponding somatic dysfunction in this section of ribs, they often report leaking droplets of milk, indicating lactogenesis I has begun.

There are numerous additional supporting ligaments of the breast that attach to osseous structures, including the clavicle, sternum, additional ribs, and scapula via the coracoid process [12]. Osteopathic techniques can be used to treat an identified somatic dysfunction of these specific bones to help release related fascial and ligamentous strains of the breast. Most lactating women find it uncomfortable to lie prone, so this position should be avoided.

The breast tissue itself can also have fascial or ligamentous restrictions that could result in pain or localized breast engorgement. Speece and Crow [14] described the fascia surrounding cells as "plastic bags." Their described treatment focused on applying a force directed to the multiple layers of these "plastic bags" to direct change [14]. Identifying and treating areas of tension within the breast itself by applying this LAS technique could potentially help with milk expression of a clogged or slow flowing lobule, reduction in local tissue edema, and a decreased viscerosomatic response. As mastitis is often preceded by a clogged milk duct or other local issue resulting in milk stasis, osteopathic manipulation of the restrictive fascia could aid in the treatment of lactational mastitis and other similar complications. Jackson and Loveless [15] reported a case using osteopathic manipulation in recurrent lactational mastitis and included myofascial release of the breast as a part of their successful treatment.

\section{Vascular}

"The rule of the artery and vein is universal in all living beings." - A.T. Still [10]

Adequate blood supply to the breast is not only vital for tissue function, but is also the delivery method for the nutrients needed that comprise breastmilk [9]. Although anatomical variants exist, the main sources of blood supply to the breast are the internal thoracic, lateral thoracic, anterior intercostal, and acromiothoracic arteries (Figure 1) [16]. Veins of the same name drain the breast and travel alongside the corresponding artery. The internal thoracic (internal mammary) artery is the most constant supplier of blood to the breast [16]. The internal thoracic artery is a branch of the subclavian artery which travels just inferior to the clavicle [17]. Therefore, the clavicle should always be examined for somatic dysfunction in a lactating patient to ensure there is no restriction that could inhibit the full flow of this artery. We commonly observe clavicular dysfunction as one of the somatic dysfunctions in patients with low milk supply and hypothesize that this could be due in part to decreased blood flow of the internal thoracic artery. Decreased blood supply is not a known cause of low milk supply but is worth considering given the known pathologic implications in many disease states that arise due to decreased tissue perfusion. 


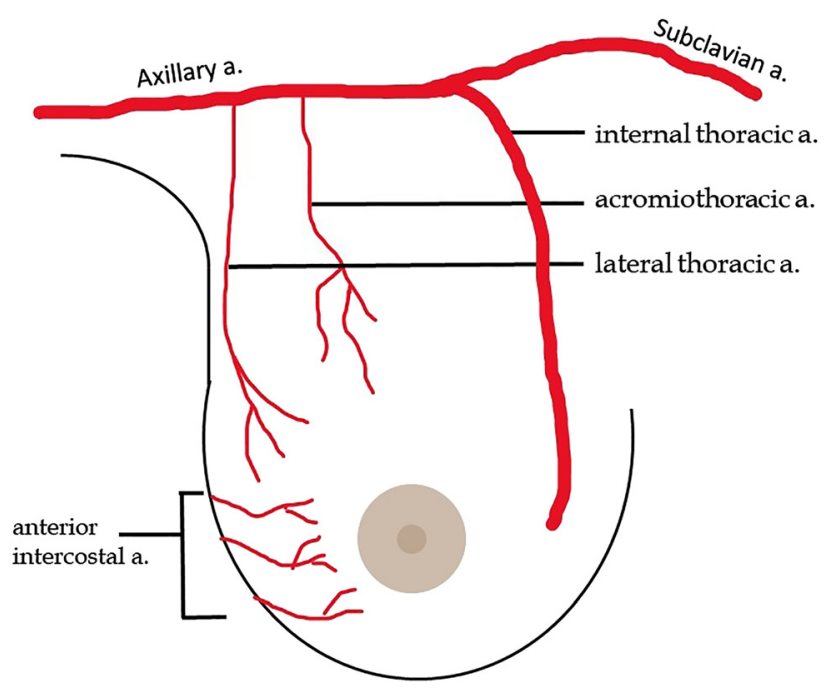

Figure 1: Blood supply of the breast.

The internal thoracic artery then descends anteromedially and is directly posterior to the pectoralis major muscle. The anterior intercostal artery is typically a single branch that then subdivides into inferior and superior branches [16]. The perforating branches of the anterior intercostal arteries pierce the pectoralis major muscle with branches two through four, providing blood supply to the breast. They naturally enlarge during lactation to accommodate the necessary increase in blood flow to the breast [16]. The lateral thoracic artery branches from the axillary artery curving around the lateral edge of pectoralis major to supply the lateral aspect of the breast [16].

While bearing down and pushing during labor, a woman in labor will often grip behind her thighs, a bedrail, or partner's hand. This can result in contraction of the pectoralis major and minor muscles. Some version of this labor practice was used in Still's time as he advised, "Be sure you have let loose the axillary and mammary veins, nerve and artery, which have been cramped by pulling the arm down during delivery." [10] The common cradling position of the infant in the mothers' arms can also result in increased tension of the pectoralis muscles. We believe releasing the tension in these muscles is key to decreasing restriction of the internal thoracic, lateral thoracic, and anterior intercostal arteries to ensure healthy blood supply to the breast. Although generally regarded as a lymphatic technique, pectoralis major restrictions can be treated simply with a pectoral traction technique [13]. Barral described his manual articular approach to specifically treat the pectoralis minor muscle, which can help release the muscular constriction around these arteries [18].

\section{Lymphatics}

"We strike at the source of life and death when we go to the lymphatics.” - A.T. Still [10]

Chikly [19] astutely observed that the breast is among the only parts of the body that does not have an inherent lymphatic pump through the routine activity. For example, walking serves as a natural lymphatic pump for the extremities and peristalsis of the intestines massages the abdominal lymph system [13, 19]. Furthermore, weeks 28-36 of pregnancy, referred to by Kuchera and Kuchera [20] as "the congestive stage," may bring accumulation of more interstitial fluid than the body can effectively remove through the lymphatic system. At this stage, the growing fetus starts to press to the upper limits of the abdominal cavity and can restrict full excursion of the diaphragm. Diaphragmatic movement is key in effective lymphatic mobilization [13]. The increased fluid accumulation and decreased ability to remove it effectively is why carpal tunnel syndrome and DeQuervian's tenosynovitis are so common in the third trimester of pregnancy [20]. The realities of breast physiology and the edematous pregnancy state can also lead to congestion in the breast, making breastfeeding in the immediate postpartum period difficult. Failure to establish good breastfeeding practices in the immediate neonatal period can have negative implications for the entire breastfeeding relationship including long-term need for supplementation, early cessation of breastfeeding, and feeding-related neonatal complications [21].

Upon transition to lactogenesis II at approximately 30-40 hours postpartum, physiologic engorgement is primarily a result of edema due to the third spacing of fluid that results from the high concentration of glucose delivered to the breast by the increased blood supply needed to nourish breastmilk [9]. Reducing the pain and discomfort associated with postpartum engorgement should therefore be focused on the reduction of edema, not the aggressive removal of milk [22]. Frequent feeding at the breast is very important, but additional pumping to increase milk extraction is usually not indicated in the absence of feeding difficulty or mother-infant separation. The mother's need to rest and increased time spent in bed recovering from labor further complicates this edema and the inherently poor lymphatic flow of the breast, which could present an opportunity for osteopathic intervention.

Although there is limited literature on the use of lymphatic techniques directed specifically at the breast, the principles of osteopathic lymphatic technique can be applied. The general approach is fourfold: "(1) remove 

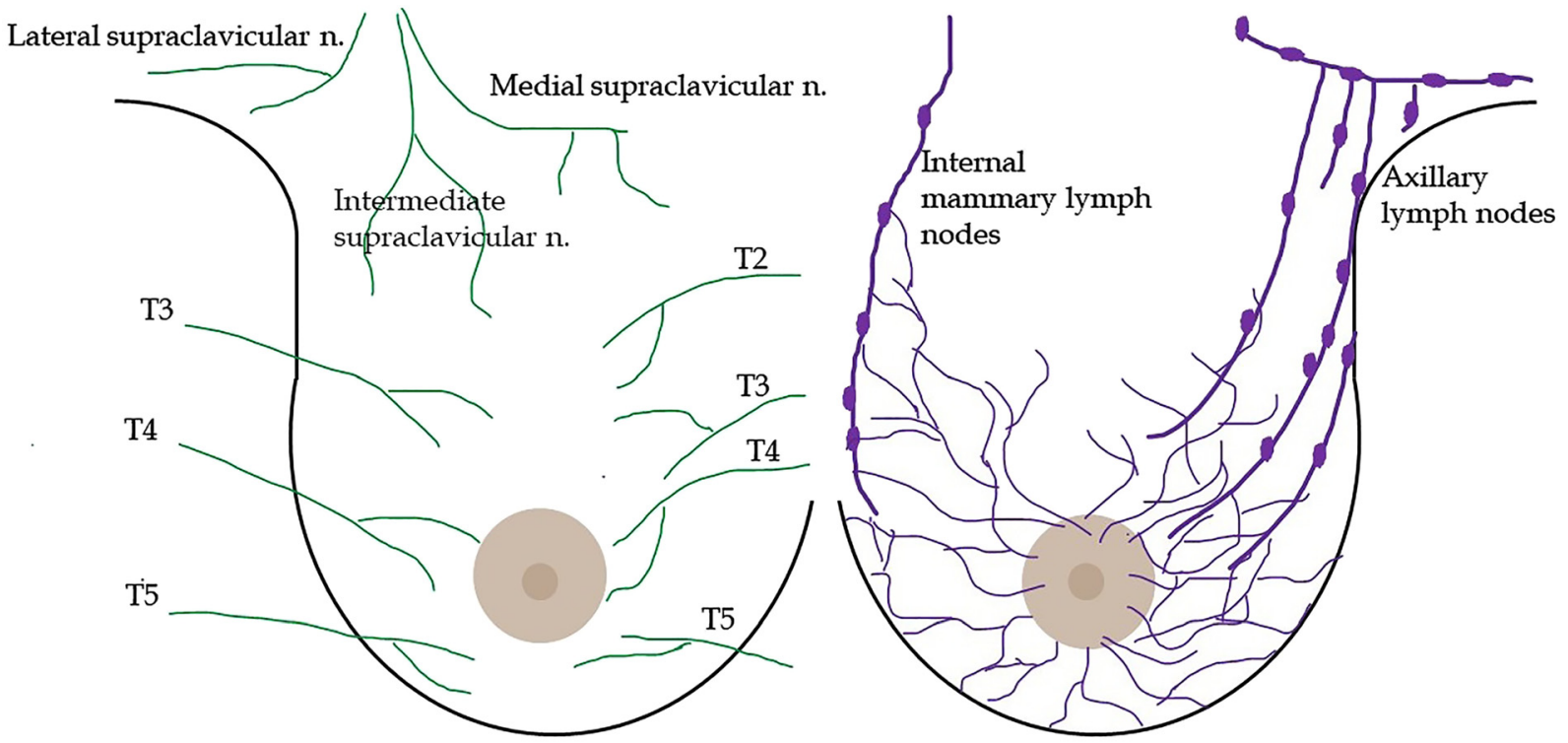

Figure 2: Breast lymphatics and innervation.

impediments to lymphatic flow; (2) enhance mechanisms involved in respiratory-circulatory homeostasis; (3) extrinsically augment the flow of lymph and other immune system elements; and (4) further mobilize lymphatic fluids from local or regional tissues that would benefit from decongestion" [13].

Given the anatomy of the breast, impediments to lymphatic flow are most likely due to fascial restrictions. To first address the respiratory-circulatory aspect of lymphostasis, any number of osteopathic techniques for the diaphragm could be utilized, including doming the diaphragm [13] and the posterior LAS approach using the 12th rib [4, 13]. After releasing the diaphragm, a lymphatic pump could be used to augment lymphatic flow. Although there is no pump described specifically for the breast, research has demonstrated that performing a lymphatic pump in one area of the body increases lymphatic flow throughout [23]. The pedal pump generally mobilizes lymphatics and also results in increased motion in the breast itself, especially if not restrained by a bra. Finally, mobilization in local and regional tissue can be addressed through effleurage, directing fluid outward away from the areola and in a slight medial-lateral direction in the inferior portion of the breast [19]. Self-lymphatic massage can be taught to the patient, instructing her to use a gentle stroking motion from the midline base of the breast up toward the axillary lymph nodes and the internal mammary nodes along the sternum (Figure 2) [24].

\section{Innervation}

"Its [the body's] nerves are so abundant that no atom of flesh fails to get nerve and fluid supply therefrom.” - A.T. Still [10]

During lactation, milk letdown occurs due to sensory nerve stimulation when the infant latches onto the nipple and starts to suck, causing a release of oxytocin [11, 25]. Oxytocin binds to the myoepithelial cells within the breast, causing contractions that push milk toward the nipple [9]. This cascade is triggered by breast sensation innervated by thoracic nerves 2 through 6 via the anterior cutaneous intercostal nerves and the lateral cutaneous intercostal nerves (Figure 2) [11, 25, 26]. Nipple sensation comes primarily from the fourth thoracic nerve (T4), although the innervation is complex, with branches of $\mathrm{T} 3$ or $\mathrm{T} 5$ assisting or solely innervating the nipple (Figure 2) [11, 26]. These sensory efferent nerves follow the corresponding rib to the spinal ganglion, which is located anterior to the rib head before entering the spinal column [26, 27]. An osteopathic physician could identify and treat any somatic dysfunction of these ribs and thoracic segments to ensure healthy breast innervation. This can be accomplished with a direct technique like high velocity, low amplitude (HVLA) or a gentler technique like rib raising or myofascial release [13].

The pituitary gland sits within the sella turcica of the sphenoid and is enveloped in dura [28]. Its hormones, including oxytocin, are released into the blood stream at the intercavernous sinus [28]. Therefore, we recommend 
that osteopathic treatment should include an examination for cranial dysfunction to ensure there is no strain around the pituitary that could inhibit full function. Treatment could include a balanced membranous tension technique to release strains in the reciprocal tension membrane or a venous sinus drainage technique to improve overall cranial function and primary respiratory mechanism $[13,29]$.

Nipple trauma is all too frequent in the postpartum mother and is most commonly due to shallow or otherwise poor infant latch [30]. A study of 1,243 mother-infant dyads found that $32 \%$ of women developed cracked nipples within 30 days of delivery [31]. This causes painful nipples and the corresponding thoracic paraspinals can become sensitized through spinal facilitation [13]. The corresponding thoracic segments should be evaluated for somatic dysfunction in effort to normalize the aberrant impulse reflex and viscerosomatic response. Similar to rib dysfunctions of the same level, we commonly observe a group somatic dysfunction involving at least T3-5 in our patients who report pain with feeding or low milk supply.

\section{Discussion}

"His mind will explore the bone, the ligament, the muscle, the fascia, the channels through which the blood travels from the heart to local destiny, with lymphatics and their contents, - the nerves the blood vessels and every channel through or over which all substances are transmitted all over the body.” - A.T. Still [10]

A careful understanding of anatomy and osteopathic principles may allow osteopathic physicians to adapt their common techniques to prevent or treat problems that may arise in lactating women. Some information may also be extrapolated from studies of other manual therapies. A 2019 systematic review of massage literature found six studies published from 1980 to 2017 related to breastfeeding [32]. The outcomes investigated in those studies included increase in breast milk supply, reduction of breast pain, resolution of blocked ducts, engorgement, mastitis, and duration of breastfeeding [32]. Therapeutic ultrasound has also been reported as a potential treatment for clogged milk ducts [33]. A retrospective case series of 25 women with a total of 34 clogged milk duct episodes while breastfeeding reported that $23(92 \%)$ of the women had resolution of breast pain and breast lump following 1-7 treatments (average, 3.3 treatments) with therapeutic ultrasound by their chiropractor [33]. There was also a 3 patient case series reporting resolution of severe, refractory breast pain during lactation after pectoralis major massage and stretching [34]. Bolman et al. [35] reported breast massage combined with hand expression as a common practice in the breastfeeding community in Russia when women present with engorgement, clogged milk ducts, or mastitis.

Gua sha, a scraping therapy used by practitioners of traditional Chinese medicine, has also been investigated for its effect on breast engorgement [36]. Its proposed mechanism is the extravasation of blood and metabolic waste to promote circulation [36]. Fifty-four women with postpartum breast engorgement were randomized to receive either gua sha therapy or hot packs and massage [36]. Subjects in the gua sha intervention group were found to have improvement $(\mathrm{p}<0.001)$ in body temperature, breast temperature, engorgement, breast pain, and breast discomfort at both the 5 and $30 \mathrm{~min}$ reassessment points following therapy [36].

\section{Limitations}

The major limitation of this article is that all mechanisms discussed here are entirely theoretical and are proposed based on a study of anatomy and examination of lactating patients in our weekly mother-baby osteopathic manipulation clinic. We suggest a possible role for osteopathic manipulation in women who experience pain with breastfeeding, engorgement, low milk supply, clogged milk ducts, and mastitis through improved innervation, blood flow, lymphatic drainage, and decrease in fascial restriction and facilitation. In line with the tenets of osteopathic medicine, when the structure is optimized, the breast should be capable of normal lactogenesis to provide both nutritive and nonnutritive benefits to the mother and the infant that long term breastfeeding affords. Using osteopathic manipulation in lactating women may not only address the mind-body unit of the mother, but that of the mother-infant dyad as well if it leads to a successful breastfeeding relationship. We are currently conducting a pilot study investigating the use of osteopathic manipulation in women with low milk supply [37]. Further studies of this and our other proposed indications are warranted.

For the sake of simplicity, the term breastfeeding is used throughout this article. This is in no way meant to marginalize populations who are more comfortable with the term chestfeeding, especially our lesbian, gay, bisexual, transgender, queer, and other patients. 


\section{Conclusions}

Osteopathic manipulation has the potential to benefit patients who are lactating, but more outcomes research is needed to establish its value. If it proves to be useful, osteopathic physicians may be uniquely poised to manage some of the challenges that breastfeeding women face and provide comprehensive care to the mother-infant dyad.

Research funding: None reported.

Author contributions: Both authors provided substantial contributions to conception and design, acquisition of data, or analysis and interpretation of data; both authors drafted the article or revised it critically for important intellectual content; both authors gave final approval of the version of the article to be published; and both authors agree to be accountable for all aspects of the work in ensuring that questions related to the accuracy or integrity of any part of the work are appropriately investigated and resolved.

Competing interests: Dr. Conaway has received speaker honoraria in the past 3 years from the AAO, ACOFP, Florida Osteopathic Medical Association, Florida Society American College of Osteopathic Family Physicians, American Academy of Family Physicians, and the Osteopathic Physicians \& Surgeons of California. She is also paid faculty of the National Board of Osteopathic Medical Examiners. Dr. O’Donnell has received speaker honoraria in the past 3 years from the Florida Osteopathic Medical Association.

\section{References}

1. Horta BL, Victora CG. Long-term effects of breastfeeding. A systematic review. The World Health Organization; 2013. http:// apps.who.int/iris/bitstream/10665/79198/1/9789241505307_ eng.pdf?ua $=1$.

2. What are the benefits of breastfeeding. National Institutes of Health. https://www.nichd.nih.gov/health/topics/breastfeeding/ conditioninfo/Pages/benefits.aspx [Accessed 15 Nov 2018].

3. van den Elsen LWJ, Garssen J, Burcelin R, Verhasselt V. Shaping the Gut microbiota by breastfeeding: the Gateway to allergy prevention? Front Pediatr 2019;7:47.

4. Schwarz EB, Nothnagle $M$. The maternal health benefits of breastfeeding. Am Fam Physician 2015;91:602-4.

5. World Health Organization. Statement: exclusive breastfeeding for six months best for babies everywhere; 2011. http://www.who.int/ mediacentre/news/statements/2011/breastfeeding_20110115/ en/.

6. American Academy of Pediatrics. Policy statement: breastfeeding and the use of human milk. Pediatrics 2012;129:e827-841.

7. Centers for Disease Control and Prevention. Breastfeeding report card; 2020. https://www.cdc.gov/breastfeeding/data/ reportcard.htm Updated Sept 17, 2020 [Accessed 23 Feb 2021].
8. Cornall D. A review of breastfeeding literature relevant to osteopathic practice. Int J Osteopath Med 2010;14:61-6.

9. Riordan J, Wambach K. Breastfeeding and human lactation, 4th ed. Sudbury, MA: Jones and Bartlett Publishers; 2010.

10. Still AT. Philosophy of osteopathy. Kirksville. MO: A.T. Still; 1899.

11. Darlington AJ. Anatomy of the breast. In: Hogg P, Kelly J, Mercer C, editors. Digital mammography: a holistic approach. Cham, Switzerland: 2015:3-10 pp.

12. Matousek S, Corlett R, Ashton M. Understanding the fascial supporting network of the breast. Plast Reconstr Surg 2014;133: 273-81.

13. American Osteopathic Association. Foundations of Osteopathic Medicine, 3rd ed. Philadelphia, PA: Lippincott Williams \& Wilkins; 2010.

14. Speece CA, Crow WT. Ligamentous articular strain. Seattle, WA: Eastland Press; 2001

15. Jackson C, Loveless B. The use of osteopathic manipulative medicine in the management of recurrent mastitis. J Am Osteopath Assoc 2020;120:921-5.

16. van Deventer PV, Graewe FR. The blood supply of the breast revisited. Plast Reconstr Surg 2016;137:1388-97.

17. Stranding S. Chest wall and breast. Gray's anatomy, 41st ed. Elsevier; 2016:931-52 pp. Chapter 53.

18. Barral JP, Croibier A. New manual articular approach. Upper Extremity. Barral Productions; 2013.

19. Chikly B. Silent waves: theory and practice of lymph drainage and therapy, 2nd ed. Scottsdale, AZ: I.H.H Publishing; 2004. Chapter 3.

20. Kuchera M, Kuchera W. Osteopathic considerations in systemic dysfunction. Columbus, OH: Greyden Press; 1994.

21. Feldman-Winter L, Kellams A, Peter-Wohl S, Taylor J, Lee K, Terrell $M$, et al. Evidence-based updates on the first week of exclusive breastfeeding among infants $\geq 35$ weeks. Pediatrics 2020;145: e20183696.

22. Berens $P$, Brodribb W. ABM clinical protocol \#20: engorgement, revised 2016. Breastfeed Med 2016;11:159-63.

23. Knott EM, Tune JD, Stoll ST, Downey HF. Increased lymphatic flow in the thoracic duct during manipulative intervention. J Am Osteopath Assoc 2005;105:447-56.

24. Witt AM, Bolman M, Kredit S. Mothers value and utilize early outpatient education on breast massage and hand expression in their self-management of engorgement. Breastfeed Med 2016;11: 433-9.

25. Hassiotou F, Geddes D. Anatomy of the human mammary gland: current status of knowledge. Clin Anat 2013;26:29-48.

26. Riccio C, Zeiderman M, Chowdhry S, Brooks R, Kelishadi S, Tutela J, et al. Plastic surgery of the breast: keeping the nipple sensitive. Eplasty 2015;15:e28.

27. Kommuru H, Jothi S, Bapuji P, Sree DL, Antony J. Thoracic part of sympathetic chain and its branching pattern variations in south Indian cadavers. J Clin Diagn Res 2014;8: AC09-AC12.

28. Mtui E, Gruener G, Dockery P. Fitzgerald's clinical neuroanatomy and neuroscience, 8th ed. Amsterdam, Netherlands: Elsevier Limited; 2021.

29. Magoun HI. Osteopathy in the cranial field, 3rd ed. Indianapolis, IN: The Cranial Academy; 1966.

30. Berens P, Eglash A, Malloy M, Steube AM. ABM clinical protocol \#26: persistent pain with breastfeeding. Breastfeed Med 2016;11: 46-53. 
31. Santos KJS, Santana GS, Vieira TO, Santos, CAST, Giugliani ERJ, Vieira GO. Prevalence and factors associated with cracked nipples in the first month postpartum. BMC Pregnan Childbirth 2016;16:209.

32. Anderson L, Kynoch K, Kildea S, Lee N. Effectiveness of breast massage for the treatment of women with breastfeeding problems: a systematic review. JBI Database System Rev Implement Rep 2019;17:1668-94.

33. Lavigne V, Gleberzon BJ. Ultrasound as a treatment of mammary blocked duct among 25 postpartum lactating women: a retrospective case series. J Chiropr Med 2012;11: 170-8.
34. Kernerman E, Park E. Severe breast pain resolved with pectoral muscle massage. J Hum Lactation 2014;30:287-91.

35. Bolman M, Saju L, Oganesyan K, Kondrashova T, Witt AM. Recapturing the art of therapeutic breast massage during breastfeeding. J Hum Lactation 2013;29:328-31.

36. Chiu JY, Gau ML, Kuo SY, Chang YH, Kuo SC, Tu HC. Effects of GuaSha therapy on breast engorgement: a randomized controlled trial. J Nurs Res 2010;18:1-10.

37. OMILQ. Osteopathic manipulation to increase lactation quantity (OMILQ). ClinicalTrials.gov Identifier: NCT03875794. https:// clinicaltrials.gov/ct2/show/NCT03875794? cond $=0$ MILQ \&draw $=2 \&$ rank $=1$. 